\title{
Complete genome analysis of African swine fever virus responsible for outbreaks in domestic pigs in 2018 in Burundi and 2019 in Malawi
}

\author{
Jean N. Hakizimana ${ }^{1,2}$. Jean B. Ntirandekura ${ }^{3}$. Clara Yona ${ }^{1,4}$ • Lionel Nyabongo . Gladson Kamwendo $^{6}$. \\ Julius L. C. Chulu ${ }^{6}$. Désiré Ntakirutimana ${ }^{5}$. Olivier Kamana ${ }^{7} \cdot$ Hans Nauwynck $^{8} \cdot$ Gerald Misinzo $^{1,2}$ (1)
}

Received: 24 April 2021 / Accepted: 30 July 2021 / Published online: 17 August 2021

(c) The Author(s) 2021

\begin{abstract}
Several African swine fever (ASF) outbreaks in domestic pigs have been reported in Burundi and Malawi and whole-genome sequences of circulating outbreak viruses in these countries are limited. In the present study, complete genome sequences of ASF viruses (ASFV) that caused the 2018 outbreak in Burundi (BUR/18/Rutana) and the 2019 outbreak in Malawi (MAL/19/Karonga) were produced using Illumina next-generation sequencing (NGS) platform and compared with other previously described ASFV complete genomes. The complete nucleotide sequences of BUR/18/Rutana and MAL/19/Karonga were 176,564 and 183,325 base pairs long with GC content of 38.62 and $38.48 \%$, respectively. The MAL/19/Karonga virus had a total of 186 open reading frames (ORFs) while the BUR/18/Rutana strain had 151 ORFs. After comparative genomic analysis, the MAL/19/Karonga virus showed greater than $99 \%$ nucleotide identity with other complete nucleotides sequences of p72 genotype II viruses previously described in Tanzania, Europe and Asia including the Georgia 2007/1 isolate. The Burundian ASFV BUR/18/Rutana exhibited 98.95 to 99.34\% nucleotide identity with genotype X ASFV previously described in Kenya and in Democratic Republic of the Congo (DRC). The serotyping results classified the BUR/18/Rutana and MAL/19/Karonga ASFV strains in serogroups 7 and 8 , respectively. The results of this study provide insight into the genetic structure and antigenic diversity of ASFV strains circulating in Burundi and Malawi. This is important in order to understand the transmission dynamics and genetic evolution of ASFV in eastern Africa, with an ultimate goal of designing an efficient risk management strategy against ASF transboundary spread.
\end{abstract}

Keywords African swine fever virus $\cdot$ Asfarviridae $\cdot$ Burundi $\cdot$ Domestic pig $\cdot$ Malawi $\cdot$ Whole-genome sequencing

\section{Introduction}

The aetiology of Africa swine fever (ASF) is ASF virus (ASFV), a linear double-stranded DNA arbovirus with a genome size ranging between 170 and 194 kilobase pairs

Gerald Misinzo

gerald.misinzo@sacids.org

1 SACIDS Africa Centre of Excellence for Infectious Diseases, SACIDS Foundation for One Health, Sokoine University of Agriculture, PO Box 3297, Morogoro, Tanzania

2 Department of Veterinary Microbiology, Parasitology and Biotechnology, College of Veterinary Medicine and Biomedical Sciences, Sokoine University of Agriculture, PO Box 3019, Morogoro, Tanzania

3 Department of Animal Health and Productions, University of Burundi, PO Box 1550, Bujumbura, Burundi (kbp), and the only member of the genus Asfivirus, family Asfarviridae (Alonso et al. 2018). However, a potential new member of the Asfarviridae family designated as Abalone asfa-like virus (AbALV) has been recently reported (Matsuyama et al. 2020). The outcome of ASF infection

4 Department of Biosciences, Solomon Mahlangu College of Science and Education, Sokoine University of Agriculture, PO Box 3038, Morogoro, Tanzania

5 National Veterinary Laboratory of Burundi, PO Box 227, Bujumbura, Burundi

6 Department of Animal Health and Livestock Development, Ministry of Agriculture, Irrigation and Water Development, PO Box 2096, Lilongwe, Malawi

7 Department of Applied Research and Development and Foresight Incubation, National Industrial Research and Development Agency, PO Box 273, Kigali, Rwanda

8 Laboratory of Virology, Faculty of Veterinary Medicine, Ghent University, Salisburylaan 133, 9820 Merelbeke, Belgium 
in domestic pigs and Eurasian wild boars depends on virulence of causative ASFV and ranges from acute to chronic disease with mortality rates approaching $100 \%$ in naïve population (Karger et al. 2019; Pikalo et al. 2019). Due to its high mortality rate, unavailability of a commercial vaccine or effective treatment, and trade restriction of domestic pigs and pork products across countries, ASF is considered as the most serious threat to the global domestic pig industry (Costard et al. 2009; Couacy-Hymann 2019; Onzere et al. 2018). Transmission of ASF is through direct contact between infected and susceptible domestic pigs or wild boars, ingestion of contaminated pork products, contact with infected fomites, indirect transmission through carcasses in the habitat in the case of wild boars, or bites by infected soft ticks of the Ornithodoros moubata complex (Chenais et al. 2018; Penrith and Vosloo 2009). Soft ticks of the $O$. moubata complex act as vectors of the ASFV while in eastern and southern Africa, asymptomatically infected wild suids mainly warthogs (Phacochoerus africanus) play an important role as ASFV reservoirs (Jori et al. 2013). The ASFV infection of other wild suids species such as bush pig (Potamochoerus larvatus) and giant forest hogs (Hylochoerus meinertzhageni) has been previously reported but their role in the epidemiology of the virus is not well known (Penrith et al. 2019).

Domestic pigs and the pig farming systems in Africa, South of the Sahara, have been reported to play an important role in the ASFV transmission and spread (Mwiine et al. 2019; Yona et al. 2020) while the high stability of ASFV in pork products is cited to be the major factor of ASFV spread across long distances. For instance, the first escape of the virus from Africa to Portugal in 1957 and again in 1960 was associated to airplane waste with contaminated pork products that was used for pig feeding while contaminated ship waste was cited to be the origin of ASFV introduction in Georgia in 2007 (Rowlands et al. 2008). More than 33 countries of Africa, South of the Sahara, have reported ASF where the disease is endemic and ASFV is becoming more prevalent in European and Asian countries threatening global food and nutritional security (Ge et al. 2019; Penrith et al. 2019).

The ASFV genome varies in size between 170 and 194 kilobase pairs ( $\mathrm{kbp}$ ) with a conserved central region of about $125 \mathrm{kbp}$, in addition to the left variable region (LVR) of 38 to $47 \mathrm{kbp}$ and the right variable region (RVR) of 13 to $16 \mathrm{kbp}$ (de Villiers et al. 2010). The variation of the genome lengths of different ASFV strains is caused by the gain or loss of members of the five different multigene families (MGF) of ASFV found in the LVR and the RVR, for instance, MGFs 100, 110, 300, 360 and 530/505 (Alonso et al. 2018). Previous studies have reported between 151 and 167 ORFs in ASFV genomes (de Villiers et al. 2010). However, an increasing number of studies have reported more than 167
ORFs in ASFV genomes especially the strains belonging to ASFV p72 genotype II, including seven Polish isolates, collected between 2016 and 2017 with 187 to 190 ORFs (Mazur-Panasiuk et al. 2019) and the ASFV strain Belgium/ Etalle/wb/2018 detected in wild boar in Belgium in 2018 with 186 ORFs (Gilliaux et al. 2019). A study that analyzed 12 complete genomes of the ASFV strains collected in Sardinia, Italy, from 1978 to 2014 reported 231 ORFs in four isolates and $235 \mathrm{ORFs}$ in eight ASFV isolates with $66 \mathrm{ORFs}$ defined as uncharacterized (Torresi et al. 2020).

Based on partial nucleotide sequence analysis of the $B 646 \mathrm{~L}$ gene that encodes for the major capsid protein $\mathrm{p} 72$, 24 (I-XXIV) ASFV genotypes have been identified and all of these have been reported to circulate in Africa, South of the Sahara (Achenbach et al. 2017; Lubisi et al. 2007; Quembo et al. 2018). Previous studies have reported ASFV p72 genotypes II, V, VIII and XII in Malawi while only ASFV p72 genotype $\mathrm{X}$ was reported in Burundi (Hakizimana et al. 2020a, b; Lubisi et al. 2005). Currently, only 3 complete and fully annotated ASFV strains belonging to p72 genotype $X$ are available in the GenBank, including two strains from Kenya and one from Democratic Republic of the Congo (DRC) (Bisimwa et al. 2021; de Villiers et al. 2010). However, despite the endemic status of ASF in Burundi, no ASFV has been fully sequenced. In addition, there is no ASFV p72 genotype II strain from Malawi that has been subjected to complete genome sequencing. In this study, we report the complete genome sequences of ASFV p72 genotype X (BUR/18/Rutana) responsible for the 2018 ASF outbreak in Burundi and ASFV 72 genotype II (MAL/19/Karonga) that caused an outbreak during 2019 in Malawi.

\section{Materials and methods}

\section{Sequencing of the ASFV complete genome}

Collection of the samples used in this study and subsequent ASF confirmation and genotyping have been previously described (Hakizimana et al. 2020a, b). Viral DNA was extracted from tissue samples using the Quick-DNA ${ }^{\mathrm{TM}}$ Miniprep Plus Kit (Zymo Research Corporation, CA, USA), following the manufacturer's instructions. Assessment of the integrity and quality of the extracted DNA was done through $1 \%$ agarose gel electrophoresis for 30 min running at $160 \mathrm{~V}$ with $0.5 \mu \mathrm{L}$ of sample DNA loaded. The starting genomic DNA for complete genome sequencing was quantified by picogreen method (Invitrogen, Catalog \# P7589) using Victor 3 fluorometry (PerkinElmer Life and Analytical Sciences, Shelton, USA). Illumina NovaSeq6000 instrument with $2 \times 150 \mathrm{bp}$ configuration was used for sequencing and TruSeq Nano DNA Kit (Catalog \# 20,015,964) was used for 
library preparation, according to the manufacturer's protocol. Quality control of the prepared library was done by 2100 Bioanalyzer using a DNA 1000 chip (Agilent Technologies, USA) while the library quantification was performed using real-time polymerase chain reaction (qPCR) according to the Illumina qPCR Quantification Protocol Guide (Catalog \# SY-930-1010). The libraries were subjected to sequencing to produce approximately 28 million paired-end reads (4 GB) per sample.

\section{Assembly and annotation of the ASFV genome}

Adapter sequences and low-quality reads trimming were performed using Trim Galore version 0.6.4 (https://www. bioinformatics.babraham.ac.uk/projects/trim_galore/) with cutadapt version 2.8 and the quality Phred score cutoff was set to 30 with a minimum reads length of 75 nucleotides. The quality of the filtered sequence data was assessed using FastQC version 0.11.9 (Andrews 2010). The quality-filtered reads were de novo assembled using SPAdes version 3.13.1 (Bankevich et al. 2012) and Megahit version 1.2.9 (Li et al. 2015). The assembly contigs were mapped to the reference genome using Burrows-Wheeler Aligner (BWA) version 0.7.17 with maximum exact match (mem) option (Li 2013) and the QUAST program version 5.0.2 (Gurevich et al. 2013) was used to evaluate the quality of the assembly. The longest overlapping scaffolds were assembled to generate the ASFV complete genomes. The Genome Annotation Transfer Utility (GATU) software (Tcherepanov et al. 2006) was used for annotation of the assembled ASFV genomes using Georgia 2007/1 (GenBank accession number NC_044959.2) and Ken05/Tk1 (GenBank accession number NC_044945.1) as reference genomes. The basic local alignment search tool for nucleotide (BLASTN) version 2.11.0 + (Zhang et al. 2000) was used for pairwise nucleotide alignment and search for nucleotide identity at GenBank nucleotide database. Multiple sequence alignment was carried out using MAFFT program version 7.221 (Katoh and Standley 2013) and the evolutionary history was inferred using the maximum likelihood method with 1000 bootstrap replications and evolutionary distances were calculated using Kimura 2-parameter model (Kimura 1980) as implemented in MEGA X (Kumar et al. 2018).

\section{Results}

\section{Characteristics of the complete genomes of Burundian and Malawian ASFV strains}

Complete genome sequences of the ASFV strains responsible for the 2018 outbreak in Rutana region, South-eastern Burundi (BUR/18/Rutana), and the 2019 outbreak in Karonga district, northern Malawi (MAL/19/Karonga), were determined in this study. The strains BUR/18/Rutana and MAL/19/Karonga belong to ASFV p72 genotypes X and II, respectively, as previously described through partial genome amplification and sequencing targeting specific genomic regions (Hakizimana et al. 2020a, b). The complete genome assembly generated genomes of $176,564 \mathrm{bp}$ for BUR/18/Rutana and 183,325 bp for MAL/19/Karonga with GC content of 38.62 and $38.48 \%$, respectively. The MAL/19/Karonga strain had a total of 186 open reading frames (ORFs) while the BUR/18/Rutana strain had 151 ORFs as highlighted by the whole-genome alignment of homologous genes between the ASFV strains described in this study and the corresponding reference genomes (Fig. 1). For MAL/19/Karonga, a total of 44 multigene family (MGF) members were identified within the genome including MGF 100 (3 members), MGF 110 (10 members), MGF 300 (3 members), MGF 360 (18 members) and MGF 505 (10 members). Furthermore, 36 MGF members were identified within the genome of BUR/18/Rutana strain including MGF 100 (1 member), MGF 110 (8 members), MGF 300 (3
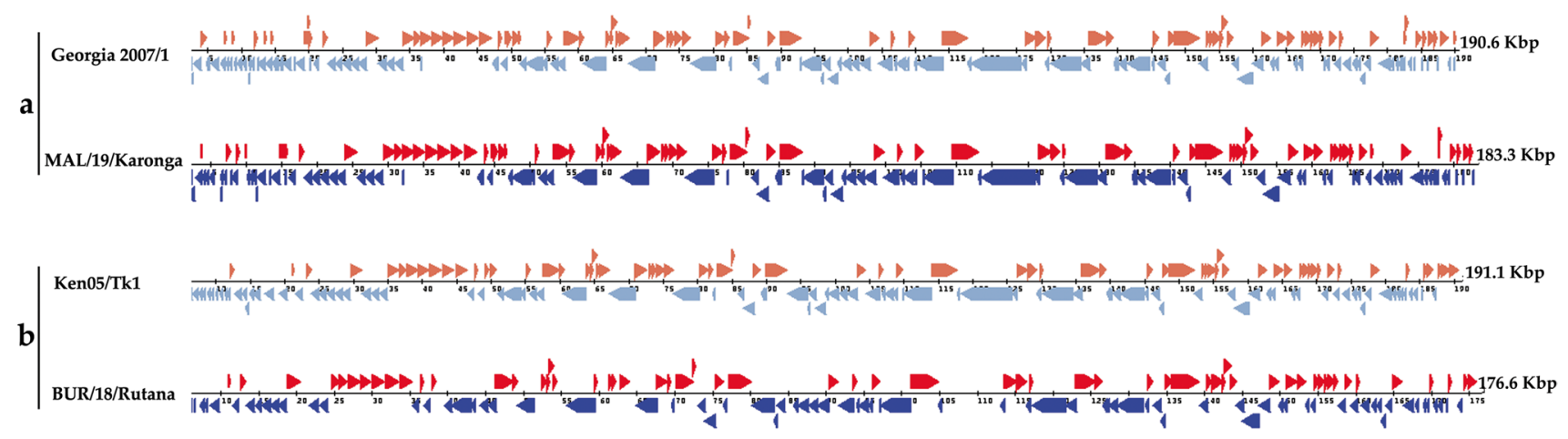

Fig. 1 Graphical display of African swine fever virus open reading frames (ORFs) of MAL/19/Karonga (a) and BUR/18/Rutana (b.) automatically annotated by Genome Annotation Transfer Utility
(GATU) using corresponding very closely related African swine fever virus reference genomes. The direction of arrows indicates the $5^{\prime}$ to 3'orientation of ORFs 
members), MGF 360 (16 members) and MGF 505 (8 members). The complete genome sequences generated in this study were submitted to GenBank and assigned accession numbers (MW856067 for BUR/18/Rutana and MW856068 for MAL/19/Karonga).

\section{Comparative genomic analysis}

Using complete genome sequences for BLASTN search at the GenBank, the MAL/19/Karonga virus was closely related to Tanzania/Rukwa/2017/1 (GenBank accession number LR813622) ASFV strain collected in South-western Tanzania from an infected domestic pig during an ASF outbreak in 2017 and belonging to ASFV p72 genotype II, with 99.97\% nucleotide identity. The percentage of nucleotide identity was greater than $99 \%$ with other complete genomic sequences of ASFV belonging to p72 genotype II isolated in Europe and Asia including the Georgia 2007/1 isolate. On the other hand, the BUR/18/Rutana ASFV strain exhibited $99.34 \%, 99.08 \%$ and $98.95 \%$ nucleotide identity with the Uvira B53 (Bisimwa et al. 2021), Ken05/Tk1 (Bishop et al. 2015) and Kenya 1950 (GenBank accession number AY261360) ASFV p72 genotype X strains, respectively (Table 1). Phylogenetic reconstruction using complete genomes clustered the MAL/19/Karonga and BUR/18/ Rutana viruses into ASFV genotypes II and X, respectively (Fig. 2). With a genome size of 183,325 bp, the MAL/19/ Karonga strain was 139 bp longer than the Tanzania/ Rukwa/2017/1 (183,186 bp) and about 6 to 7 kbp shorter than some ASFV p72 genotype II isolates available in the GenBank nucleotide database, for instance Georgia 2007/1 (190,584 bp), Arm/07/CBM/c2 (190,145 bp) and ASFVwbBS01 (189,394 bp). Furthermore, the BUR/18/Rutana strain with the genome length of 176,564 bp was about 4 to $17 \mathrm{kbp}$ shorter than the Uvira B53 (180,916 bp), Ken05/ Tk1 (191,058 bp) and Kenya 1950 (193,886 bp) ASFV p72 genotype $\mathrm{X}$ genomes. The difference in genome length is due to differences within some genes and MGF members. For instance, among the 186 ORFs identified in MAL/19/ Karonga, 151 ORFs had $100 \%$ nucleotide identity with their homologues in the reference genome while the remaining 35 ORFs were polymorphic with nucleotide identity with the reference genome varying from 60 to $99.9 \%$. In addition, four ORFs (MGF 360-1Lb, ASFV G ACD 00,120, MGF 110-7L and MGF 110-10-L-MGF110-14L fusion) present in ASFV reference genome were below the $60 \%$ nucleotide identity threshold and considered absent in the MAL/19/ Karonga virus. Among the 151 ORFs identified in BUR/18/ Rutana virus, only 35 ORFs had $100 \%$ nucleotide identity with their homologues in Ken05/Tk1 reference genome while the remaining ones had between 60 and $99.9 \%$ identity. Besides, 10 MGF members (MGF 110-7L, MGF 1108L, MGF 100-1R, MGF 110-9L, MGF 110-11L (FRAG-2),
MGF 110-13L-14L, MGF 360-12L, MGF 360-15R, MGF 100-3L, MGF 360-18R) present in the Ken05/Tk1 reference genome had a nucleotide similarity below $60 \%$ compared to their homologues in BUR/18/Rutana virus.

\section{Determination of the serogroups of Burundian and Malawian ASFV strains based on EP402R (CD2v) gene sequences}

In order to classify the ASFV strains described in this study among the eight previously determined serogroups based on the ASFV hemadsorption inhibition (HAI) properties, we compared sequences of the $E P 402 R$ gene that encodes the $\mathrm{CD} 2 \mathrm{v}$ major ASFV antigen protein between them and selected isolates representing each serogroup retrieved from GenBank. A high nucleotide sequence variation was observed among the compared sequences and the serotyping results classified the BUR/18/Rutana and MAL/19/Karonga ASFV viruses in serogroups 7 and 8, respectively (Fig. 3). The Burundian ASFV strain grouped together with two strains belonging to serogroup 7 previously described, for instance the Uvira B53 ASFV strain collected during an ASF outbreak in South Kivu province of the Democratic Republic of the Congo (DRC) in 2019 and the Uganda ASFV strain (Bisimwa et al. 2021; Malogolovkin et al. 2015a, b), whereas the MAL/19/Karonga ASFV strain clustered together with strains belonging to ASFV serogroup 8 previously described in Europe and Asia.

\section{Discussion}

The limited knowledge of the genetic variation of the ASFV has hindered the development of effective control and prevention strategies, including vaccine, diagnostic test and antiviral treatment development (Arabyan et al. 2019; Bao et al. 2021; Torresi et al. 2020; Urbano et al. 2021). Partial nucleotide sequencing of specific ASFV genomic regions is conventionally used to determine ASFV genotypes and to discriminate closely related ASFV strains. However, in order to obtain adequate information on transmission dynamics, genetic variation and molecular evolution of different ASFV strains, complete genome sequencing is required. To date, despite the regular reports of the ASFV p72 genotype II in different countries of eastern and southern Africa, only one fully annotated complete genome of the genotype II from those countries is publicly available, for instance the Tanzania/Rukwa/2017/1 collected in South-western Tanzania from an infected domestic pig during an outbreak in 2017 (Njau et al. 2021). There is no ASFV p72 genotype II strain from Malawi that has been subjected to complete genome sequencing and no ASFV strain from Burundi that has been fully sequenced. In the present study, complete genome 


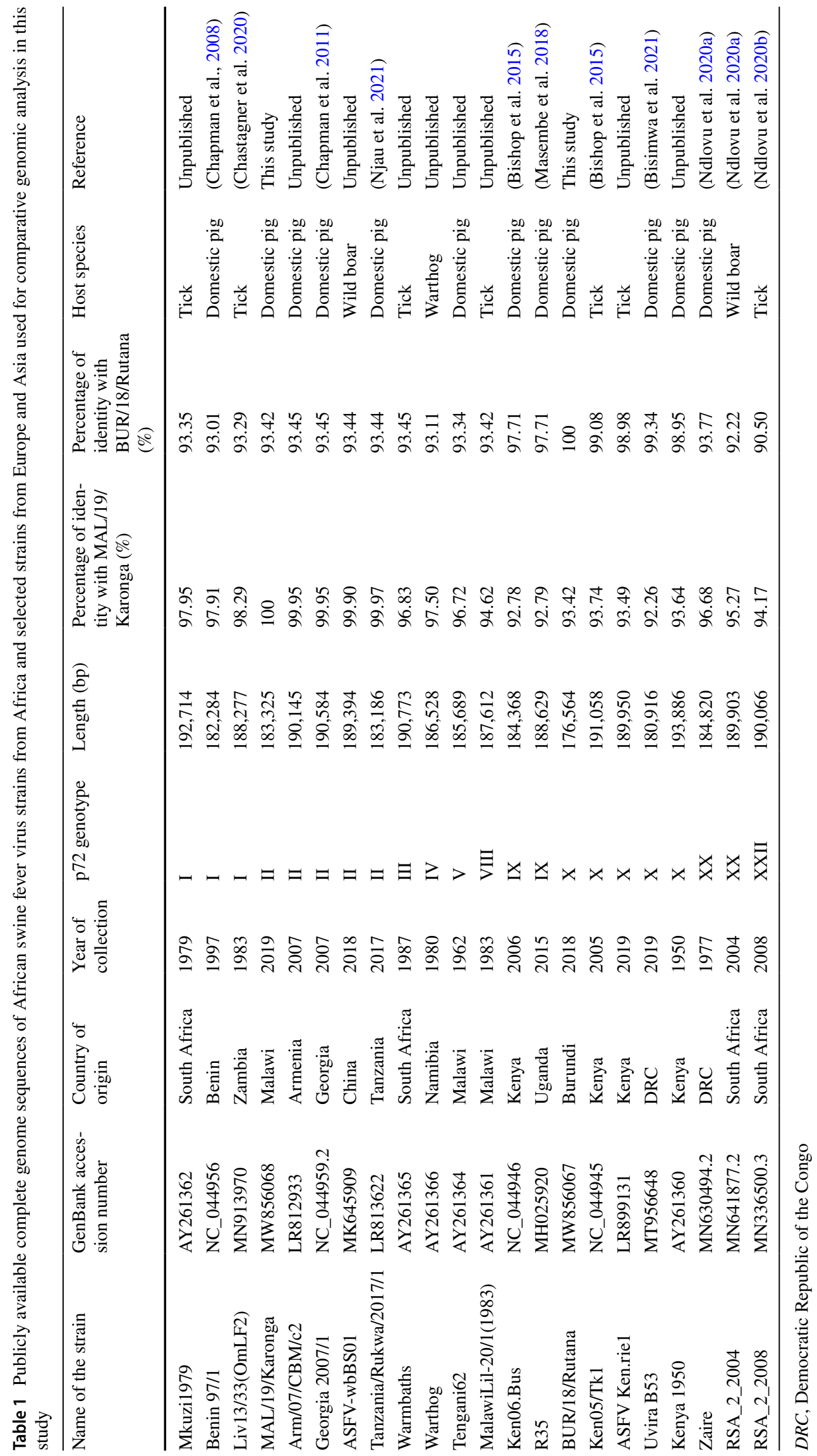


Fig. 2 Maximum likelihood phylogenetic tree obtained after multiple sequence alignment of complete genomes of African swine fever virus strains from Africa and selected strains from Europe and Asia. The viruses described in this study are indicated by black squares and the scale bar indicates nucleotide substitution per site while the node values show percentage of bootstrap support. The analysis involved 22 nucleotide sequences with a total of 166,578 positions in the final dataset

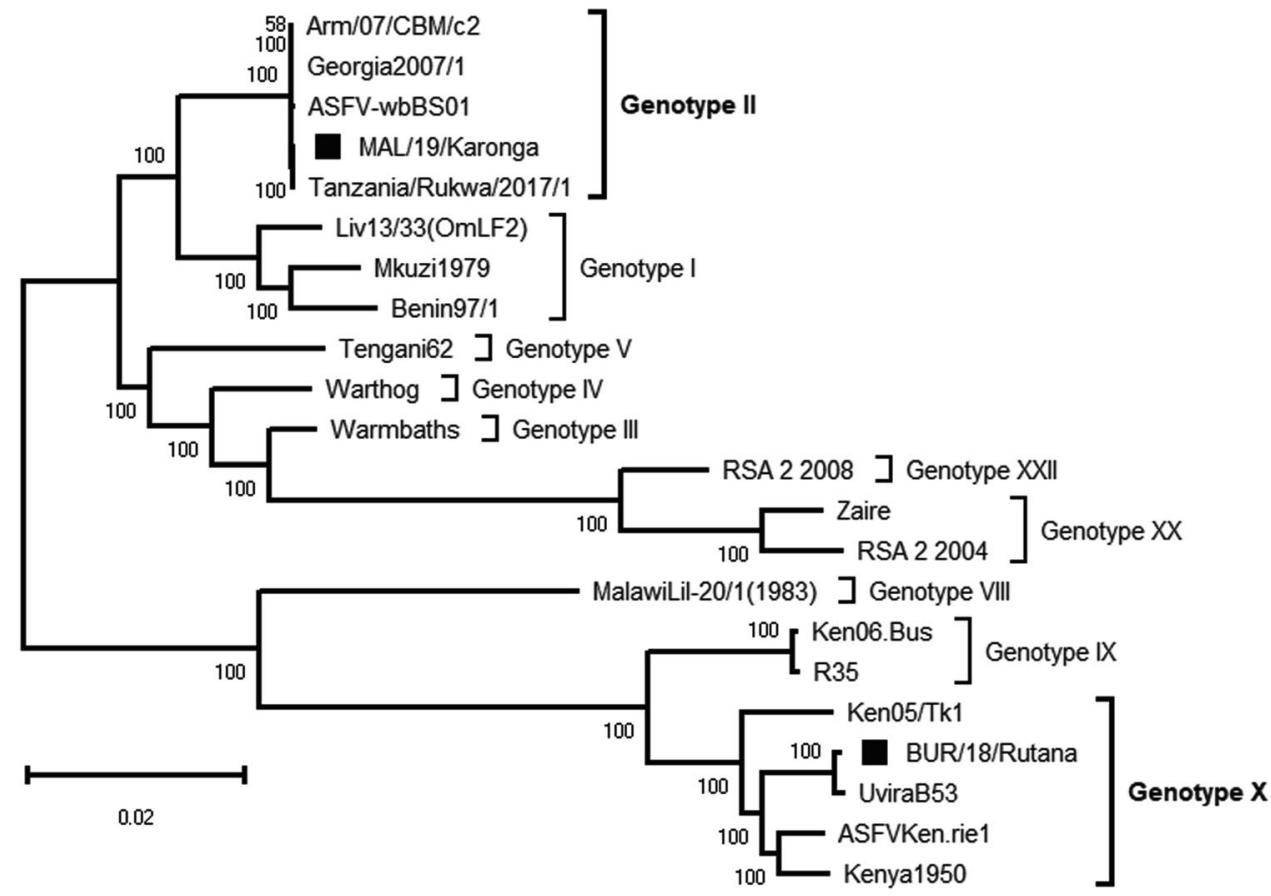

sequences of the ASFV p72 genotype X responsible for the 2018 outbreak in Burundi and genotype II virus that caused the 2019 ASF outbreak in Malawi were generated using Illumina NGS technology. The complete genome sequences generated in this study were closely related to ASFV strains previously described, available in the GenBank database, belonging to ASFV p72 genotype X for the BUR/18/Rutana strain from Burundi and to genotype II for the MAL/19/ Karonga strain from Malawi. Besides, serotyping results classified the BUR/18/Rutana and MAL/19/Karonga ASFV strains into ASFV serogroups 7 and 8, respectively.

The Burundian ASFV strain was more closely related to Uvira B53 ASFV strain collected during an ASF outbreak in in South Kivu province of the DRC (Bisimwa et al. 2021), with $99.34 \%$ nucleotides identity. These findings are in agreement with the results of studies using partial nucleotide sequencing where relatedness between those two ASFV strains were reported (Bisimwa et al. 2020; Hakizimana et al. 2020b) highlighting the possibility of transboundary spread of genotype $\mathrm{X}$ viruses between
Burundi and DRC, as previously speculated. Furthermore, the Malawian ASFV strain described in this study was more closely related to the Tanzania/Rukwa/2017/1 ASFV strain collected in South-western Tanzania from an infected domestic pig during an ASF outbreak in 2017 (Njau et al. 2021), with $99.97 \%$ nucleotide identity. The high nucleotide similarity between ASFV p72 genotype II strains circulating in Malawi and Tanzania has been previously reported by studies using partial nucleotide sequencing suggesting a common source and transboundary spread of ASFV between these two countries (Hakizimana et al. 2020a; Misinzo et al. 2012). In addition, the Malawian ASFV strain had more than $99 \%$ nucleotides identity with ASFV p72 genotype II viruses previously described in Europe and Asia suggesting a possible common ancestor of these ASFV strains as previously speculated (Hakizimana et al. 2020a; Misinzo et al. 2012; Quembo et al. 2018; Rowlands et al. 2008).

Comparative genomic analysis revealed genetic variation in the ASFV strains described in this study compared to
Fig. 3 A maximum likelihood phylogenetic tree of the ASFV $E P 402 R(\mathrm{CD} 2 \mathrm{v})$ gene indicating serogroups of selected ASFV strains. The strains responsible for the 2018 and 2019 ASF outbreak in Burundi and in Malawi are indicated by a black dot. The scale bar indicates the number of substitutions per site

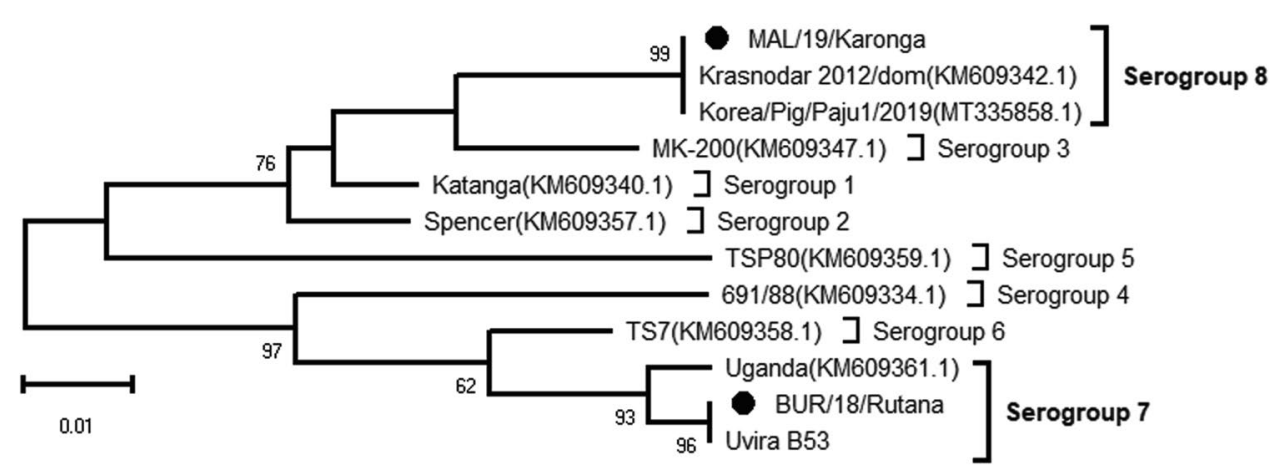


ASFV genomes previously described available in the GenBank. For instance, the DP96R gene reported as absent in the Uvira B53 ASFV strain was present in BUR/18/Rutana and MAL/19/Karonga strains with $93.6 \%$ and $100 \%$ nucleotide identity with the Ken05/Tk1 ASFV p72 genotype X and Georgia 2007/1 ASFV p72 genotype II reference genomes, respectively. The DP96R gene encodes the UK protein potentially involved in determining the ASFV virulence in domestic pigs (Zsak et al. 1998) and its presence in BUR/18/ Rutana and MAL/19/Karonga ASFV strains may explain the high virulence of these strains as evidenced by high mortality rate during the 2018 and 2019 ASF outbreaks in Rutana region of Burundi and Karonga district in northern Malawi, as previously described (Hakizimana et al. 2020a, b). In addition, the K196R and the B119L (9GL) genes encoding the thymidine kinase and sulfhydryl oxidase enzymes, respectively, also described as the factors of virulence for ASFV (Rodríguez et al. 2015) were present in BUR/18/ Rutana and MAL/19/Karonga ASFV strains.

Previous studies have reported important genetic variation within the members of the MGFs located at the both ends of the ASFV genome resulting in difference of the genome size of different ASFV strains (Torresi et al. 2020; Urbano et al. 2021). In the present study, several single-nucleotide polymorphisms (SNPs) and complete ORF deletion were observed within different MGF members. For instance, four MGF members (MGF 100-1R, MGF 110-7L, MGF 110$8 \mathrm{~L}$ and MGF 110-9L) absent in the BUR/18/Rutana strains were also missing in the Uvira B53 strains as previously reported (Bisimwa et al. 2021). The MGF 360-1Lb gene was truncated in the MAL/19/Karonga strain and the same observation was reported in China/2018/AnhuiXCGQ ASFV strain collected during an ASF outbreak in domestic pigs in Anhui province of China in September 2018 (Bao et al. 2019). In addition, a deletion of almost all members of the MGF 110 were reported in the Estonia 2014 ASFV strain (Zani et al. 2018). The impact of these genetic variations on the phenotypes of the ASFV strains described in this study is subject to further investigations.

The protein $\mathrm{pEP} 402 \mathrm{R}$, a homologue of the T-lymphocyte surface antigen $\mathrm{CD} 2$, encoded by the $E P 402 R$ gene is located in the lipoprotein membrane of the outer viral envelope and plays an important role in the adhesion of erythrocytes to infected cells (hemadsorption) and the binding of the ASFV particles to host erythrocytes during infection (Alejo et al. 2018; Dixon et al. 2019). This gene has been used to define eight viral antigenic types called serogroups (Malogolovkin and Kolbasov 2019). The results of the present study showed that the BUR/18/Rutana and MAL/19/ Karonga ASFV strains may share the hemadsorption properties with ASFV strains belonging to serogroups 7 and 8, respectively (Fig. 3). It has been reported that the ASFV isolates classified into the same serotype show cross-protection responses from challenge during the vaccine development experiments (Malogolovkin et al. 2015a, b; Sánchez et al. 2019). Thus, the determination of the ASFV serogroups was suggested as a perfect tool for discriminating ASFV strains with different virulence and prediction of the efficacy of a specific ASFV vaccine (Burmakina et al. 2016). Recently, genetic signatures specific to each ASFV serotype have been described with the potential of elucidating more on the genetic and antigenic diversity of the ASFV (Malogolovkin et al. 2020; Urbano et al. 2021). Interestingly, the ASFV strains described in this study had the PPPKPC amino acid sequences repeated 4 and 3 times in the BUR/18/Rutana and MAL/19/Karonga ASFV strains, respectively. Similar tandem amino acid repeat sequences within the EP402R $(\mathrm{CD} 2 \mathrm{v})$ gene were reported in the Uvira B53 ASFV strain (Bisimwa et al. 2021).

In conclusion, the results of this study provided important insight into the genetic structure of the ASFV p72 genotype $\mathrm{X}$ responsible for the 2018 outbreak in Burundi and genotype II virus that caused the 2019 ASF outbreak in Malawi. Additionally, the strains BUR/18/Rutana and MAL/19/ Karonga were classified into ASFV serogroups 7 and 8 , respectively. These results will serve as backbone for possible future investigations concerning molecular evolution, transmission dynamics, diagnostic improvement and control strategies for ASFV.

Acknowledgements The authors are very grateful to Mariam R. Makange, Mhoja Ndalahwa and Anna Rogath for their excellent technical assistance during the laboratory analysis.

Author contribution Conceptualization: J.H., G.M., H.N., O.K. and J.N.; sample collection: J.H., L.N., G.K., D.N., and J.C.; methodology: J.H., G.M., C.Y. and H.N.; formal analysis: J.H., C.Y. and G.M.; data curation: J.H. and C.Y.; writing — original draft preparation: J.H. and G.M.; writing — review and editing: G.M., H.N., O.K., J.N., L.N., D.N., J.C., G.K. and C.Y. All authors read and approved the final manuscript.

Funding This work was supported by the Partnership for Skills in Applied Sciences, Engineering and Technology (PASET) through the Regional Scholarship and Innovation Fund (RSIF) awarded to J.N.H to carry out doctoral studies at SACIDS Africa Centre of Excellence for Infectious Diseases, SACIDS Foundation for One Health, Sokoine University of Agriculture, Morogoro, Tanzania. J.N.H. is also recipient of the research grant (grant number B_6269-1) from the International Foundation for Sciences (IFS) to study the epidemiology of African swine fever in countries neighboring Tanzania.

Availability of data and material The nucleotide sequences generated in the present study were submitted to the NCBI GenBank with accession numbers MW856067 and MW856068.

\section{Declarations}

The samples used in this study were collected from naturally dead domestic pigs according to common veterinary practice as part of routine veterinary investigation in Burundi and Malawi. In Burundi, sample collection was done according to the Burundian Animal Health Law (Law No. 1/28 of 24th December 2009) and the Law No. 1/06 of 21st 
March 2011 related to the practice of veterinary medicine in Burundi while in Malawi, the Control and Diseases of Animals Act (CAP 66:02 of 1967) and the rule 6 of the Swine Fever Rules G.N. 209/1968 were followed. Oral consent was obtained from the domestic pig owners before sampling of their dead domestic pigs and documented in the Veterinary Officer registry.

Conflict of interest The authors declare no competing interests.

Disclaimer The funder had no role in study design, data collection and analysis, decision to publish and in the preparation of this manuscript.

Open Access This article is licensed under a Creative Commons Attribution 4.0 International License, which permits use, sharing, adaptation, distribution and reproduction in any medium or format, as long as you give appropriate credit to the original author(s) and the source, provide a link to the Creative Commons licence, and indicate if changes were made. The images or other third party material in this article are included in the article's Creative Commons licence, unless indicated otherwise in a credit line to the material. If material is not included in the article's Creative Commons licence and your intended use is not permitted by statutory regulation or exceeds the permitted use, you will need to obtain permission directly from the copyright holder. To view a copy of this licence, visit http://creativecommons.org/licenses/by/4.0/.

\section{References}

Achenbach, J.E., Gallardo, C., Nieto-Pelegrín, E., Rivera-Arroyo, B., Degefa-Negi, T., Arias, M., Jenberie, S., Mulisa, D.D., Gizaw, D., Gelaye, E., Chibssa, T.R., Belaye, A., Loitsch, A., Forsa, M., Yami, M., Diallo, A., Soler, A., Lamien, C.E., Sánchez-Vizcaíno, J.M., 2017. Identification of a New Genotype of African Swine Fever Virus in Domestic Pigs from Ethiopia. Transboundary and Emerging Diseases 64, 1393-1404. https://doi.org/10.1111/tbed. 12511

Alejo, A., Matamoros, T., Guerra, M., Andrés, G., 2018. A Proteomic Atlas of the African Swine Fever Virus Particle. Journal of Virology 92, e01293-18. https://doi.org/10.1128/JVI.01293-18

Alonso, C., Borca, M., Dixon, L., Revilla, Y., Rodriguez, F., Escribano, J.M., ICTV Report Consortium, 2018. ICTV Virus Taxonomy Profile: Asfarviridae. Journal of General Virology 99, 613-614. https://doi.org/10.1099/jgv.0.001049

Andrews, S., 2010. FastQC A Quality Control tool for High Throughput Sequence Data. Babraham Bioinformatics. https://www.bioin formatics.babraham.ac.uk/projects/fastqc/ (accessed 7 Jun 2020).

Arabyan, E., Kotsynyan, A., Hakobyan, A., Zakaryan, H., 2019. Antiviral agents against African swine fever virus. Virus Research 270, 197669. https://doi.org/10.1016/j.virusres.2019.197669

Bankevich, A., Nurk, S., Antipov, D., Gurevich, A.A., Dvorkin, M., Kulikov, A.S., Lesin, V.M., Nikolenko, S.I., Pham, S., Prjibelski, A.D., Pyshkin, A.V., Sirotkin, A.V., Vyahhi, N., Tesler, G., Alekseyev, M.A., Pevzner, P.A., 2012. SPAdes: A New Genome Assembly Algorithm and Its Applications to Single-Cell Sequencing. Journal of Computational Biology 19, 455-477. https://doi. org $/ 10.1089 / \mathrm{cmb} .2012 .0021$

Bao, J., Wang, Q., Lin, P., Liu, C., Li, L., Wu, X., Chi, T., Xu, T., Ge, S., Liu, Y., Li, J., Wang, S., Qu, H., Jin, T., Wang, Z., 2019. Genome comparison of African swine fever virus China/2018/ AnhuiXCGQ strain and related European p72 Genotype II strains. Transboundary and Emerging Diseases 66, 1167-1176. https:// doi.org/10.1111/tbed.13124
Bao, Y.-J., Qiu, J., Luo, Y., Rodríguez, F., Qiu, H.-J. 2021.The genetic variation landscape of African swine fever virus reveals frequent positive selection and adaptive flexibility. Transboundary and Emerging Diseases n/a.https://doi.org/10.1111/tbed.14018

Bishop, R.P., Fleischauer, C., de Villiers, E.P., Okoth, E.A., Arias, M., Gallardo, C., Upton, C., 2015. Comparative analysis of the complete genome sequences of Kenyan African swine fever virus isolates within p72 genotypes IX and X. Virus Genes 50, 303-309. https://doi.org/10.1007/s11262-014-1156-7

Bisimwa, P.N., Ongus, J.R., Tiambo, C.K., Machuka, E.M., Bisimwa, E.B., Steinaa, L., Pelle, R., 2020. First detection of African swine fever (ASF) virus genotype $\mathrm{X}$ and serogroup 7 in symptomatic pigs in the Democratic Republic of Congo. Virology Journal 17, 135. https://doi.org/10.1186/s12985-020-01398-8

Bisimwa, P.N., Ongus, J.R., Steinaa, L., Bisimwa, E.B., Bochere, E., Machuka, E.M., Entfellner, J.-B.D., Okoth, E., Pelle, R., 2021. The first complete genome sequence of the African swine fever virus genotype $\mathrm{X}$ and serogroup 7 isolated in domestic pigs from the Democratic Republic of Congo. Virology Journal 18, 23. https://doi.org/10.1186/s12985-021-01497-0

Burmakina, G., Malogolovkin, A., Tulman, E., Zsak L, Delhon G., Diel D., Shobogorov N., Morgunov Y., Morgunov S., Kutish G., Kolbasov D., Rock D., 2016. African swine fever virus serotypespecific proteins are significant protective antigens for African swine fever. The Journal of General Virology 97.https://doi.org/ 10.1099/jgv.0.000490

Chapman, D.A.G., Tcherepanov, V., Upton, C., Dixon, L.K., 2008. Comparison of the genome sequences of nonpathogenic and pathogenic African swine fever virus isolates. J. Gen. Virol. 89, 397-408. https://doi.org/10.1099/vir.0.83343-0

Chapman, D.A.G., Darby, A.C., Da Silva, M., Upton, C., Radford, A.D., Dixon, L.K., 2011. Genomic analysis of highly virulent Georgia 2007/1 isolate of African swine fever virus. Emerging Infectious Diseases 17, 599-605. https://doi.org/10.3201/eid17 04.101283

Chastagner, A., Pereira de Oliveira, R., Hutet, E., Le Dimna, M., Paboeuf, F., Lucas, P., Blanchard, Y., Dixon, L., Vial, L., Le Potier, M.-F., 2020. Coding-Complete Genome Sequence of an African Swine Fever Virus Strain Liv13/33 Isolate from Experimental Transmission between Pigs and Ornithodoros moubata Ticks. Microbiol. Resour. Announc. 9. https://doi.org/10.1128/ MRA.00185-20

Chenais, E., Ståhl, K., Guberti, V., Depner, K., 2018. Identification of Wild Boar-Habitat Epidemiologic Cycle in African Swine Fever Epizootic. Emerging Infectious Diseases journal 24, 810-812. https://doi.org/10.3201/eid2404.172127

Costard, S., Wieland, B., de Glanville, W., Jori, F., Rowlands, R., Vosloo, W., Roger, F., Pfeiffer, D.U., Dixon, L.K., 2009. African swine fever: how can global spread be prevented? Philos. Trans. R. Soc. B Biol. Sci. 364, 2683-2696. https://doi.org/10.1098/rstb. 2009.0098

Couacy-Hymann, E., 2019. African Swine Fever in Sub-Saharan African Countries, in: Kardjadj, M., Diallo, A., Lancelot, R. (Eds.), Transboundary Animal Diseases in Sahelian Africa and Connected Regions. Springer International Publishing, Cham, pp. 323-344. https://doi.org/10.1007/978-3-030-25385-1_16

de Villiers, E.P., Gallardo, C., Arias, M., da Silva, M., Upton, C., Martin, R., Bishop, R.P., 2010. Phylogenomic analysis of $11 \mathrm{com}-$ plete African swine fever virus genome sequences. Virology 400, 128-136. https://doi.org/10.1016/j.virol.2010.01.019

Dixon, L.K., Islam, M., Nash, R., Reis, A.L., 2019. African swine fever virus evasion of host defences. Virus Research 266, 25-33. https:// doi.org/10.1016/j.virusres.2019.04.002

Ge, S., Liu, Y., Li, L., Wang, Q., Li, J., Ren, W., Liu, C., Bao, J., Wu, X., Wang, Z., 2019. An extra insertion of tandem repeat sequence 
in African swine fever virus, China, 2019. Virus Genes 55, 843847. https://doi.org/10.1007/s11262-019-01704-9

Gilliaux, G., Garigliany, M., Licoppe, A., Paternostre, J., Lesenfants, C., Linden, A., Desmecht, D., 2019. Newly emerged African swine fever virus strain Belgium/Etalle/wb/2018: Complete genomic sequence and comparative analysis with reference $\mathrm{p} 72$ genotype II strains. Transboundary and Emerging Diseases 66, 2566-2591. https://doi.org/10.1111/tbed.13302

Gurevich, A., Saveliev, V., Vyahhi, N., Tesler, G., 2013. QUAST: quality assessment tool for genome assemblies. Bioinformatics 29 , 1072-1075. https://doi.org/10.1093/bioinformatics/btt086

Hakizimana, J. N., Kamwendo, G., Chulu, J.L.C., Kamana, O., Nauwynck, H.J., Misinzo, G., 2020a. Genetic profile of African swine fever virus responsible for the 2019 outbreak in northern Malawi. BMC Veterinary Research 16, 316. https://doi.org/10. 1186/s12917-020-02536-8

Hakizimana, Jean N., Nyabongo, L., Ntirandekura, J.B., Yona, C., Ntakirutimana, D., Kamana, O., Nauwynck, H., Misinzo, G., 2020b. Genetic Analysis of African Swine Fever Virus From the 2018 Outbreak in South-Eastern Burundi. Frontiers Veterinary Sciences 7.https://doi.org/10.3389/fvets.2020.578474

Hakizimana, J.N., Yona, C., Kamana, O., Nauwynck, H., Misinzo, G., 2021. African Swine Fever Virus Circulation between Tanzania and Neighboring Countries: A Systematic Review and Meta-Analysis. Viruses 13, 306. https://doi.org/10.3390/v1302 0306

Jori, F., Vial, L., Penrith, M.L., Pérez-Sánchez, R., Etter, E., Albina, E., Michaud, V., Roger, F., 2013. Review of the sylvatic cycle of African swine fever in sub-Saharan Africa and the Indian ocean. Virus Research 173, 212-227. https://doi.org/10.1016/j. virusres.2012.10.005

Karger, A., Pérez-Núñez, D., Urquiza, J., Hinojar, P., Alonso, C., Freitas, F.B., Revilla, Y., Le Potier, M.-F., Montoya, M., 2019. An Update on African Swine Fever Virology. Viruses 11.https:// doi.org/10.3390/v11090864

Katoh, K., Standley, D.M., 2013. MAFFT Multiple Sequence Alignment Software Version 7: Improvements in Performance and Usability. Molecular Biology and Evolution 30, 772-780. https://doi.org/10.1093/molbev/mst010

Kimura, M., 1980. A simple method for estimating evolutionary rates of base substitutions through comparative studies of nucleotide sequences. Journal of Molecular Evolution 16, 111-120. https:// doi.org/10.1007/BF01731581

Kim, H.-J., Cho, K.-H., Ryu, J.-H., Jang, M.-K., Chae, H.-G., Choi, J.-D., Nah, J.-J., Kim, Y.-J., Kang, H.-E., 2020. Isolation and Genetic Characterization of African Swine Fever Virus from Domestic Pig Farms in South Korea, 2019.Viruses 12.https:// doi.org/10.3390/v12111237

Kumar, S., Stecher, G., Li, M., Knyaz, C., Tamura, K., 2018. MEGA $\mathrm{X}$ : Molecular Evolutionary Genetics Analysis across Computing Platforms. Molecular Biology and Evolution 35, 1547-1549. https://doi.org/10.1093/molbev/msy096

Li, H., 2013. Aligning sequence reads, clone sequences and assembly contigs with BWA-MEM. ArXiv13033997 Q-Bio.

Li, D., Liu, C.-M., Luo, R., Sadakane, K., Lam, T.-W., 2015. MEGAHIT: an ultra-fast single-node solution for large and complex metagenomics assembly via succinct de Bruijn graph. Bioinformatics (Oxford, England) 31, 1674-1676. https://doi.org/10.1093/ bioinformatics/btv033

Lubisi, B.A., Bastos, A.D.S., Dwarka, R.M., Vosloo, W., 2005. Molecular epidemiology of African swine fever in East Africa. Archives of Virology 150, 2439-2452. https://doi.org/10.1007/ s00705-005-0602-1

Lubisi, B.A., Bastos, A.D.S., Dwarka, R.M., Vosloo, W., 2007. Intragenotypic resolution of African swine fever viruses from an East
African domestic pig cycle: a combined p72-CVR approach. Virus Genes 35, 729-735. https://doi.org/10.1007/s11262-007-0148-2

Malogolovkin, A., Kolbasov, D., 2019. Genetic and antigenic diversity of African swine fever virus. Virus Research 271, 197673. https:// doi.org/10.1016/j.virusres.2019.197673

Malogolovkin, Alexander, Burmakina, G., Titov, I., Sereda, A., Gogin, A., Baryshnikova, E., Kolbasov, D., 2015a. Comparative analysis of African swine fever virus genotypes and serogroups. Emerging Infectious Diseases 21, 312-315. https://doi.org/10.3201/eid2102. 140649

Malogolovkin, A., Burmakina, G., Tulman, E.R., Delhon, G., Diel, D.G., Salnikov, N., Kutish, G.F., Kolbasov, D., Rock, D.L., 2015b. African swine fever virus CD2v and C-type lectin gene loci mediate serological specificity. Journal of General Virology 96, 866873. https://doi.org/10.1099/jgv.0.000024

Malogolovkin, A., Sereda, A., Kolbasov, D., 2020. African Swine Fever Virus, in: Malik, Y.S., Singh, R.K., Yadav, M.P. (Eds.), Emerging and Transboundary Animal Viruses, Livestock Diseases and Management. Springer, Singapore, pp. 27-53. https://doi.org/ 10.1007/978-981-15-0402-0_2

Masembe, C., Sreenu, V.B., Filipe, A.D.S., Wilkie, G.S., Ogweng, P., Mayega, F.J., Muwanika, V.B., Biek, R., Palmarini, M., Davison, A.J., 2018. Genome Sequences of Five African Swine Fever Virus Genotype IX Isolates from Domestic Pigs in Uganda. Microbiology Resource Announcements 7, e01018-18. https://doi.org/10. 1128/MRA.01018-18

Matsuyama, T., Takano, T., Nishiki, I., Fujiwara, A., Kiryu, I., Inada, M., Sakai, T., Terashima, S., Matsuura, Y., Isowa, K., Nakayasu, C., 2020. A novel Asfarvirus-like virus identified as a potential cause of mass mortality of abalone. Scientific Reports 10, 4620. https://doi.org/10.1038/s41598-020-61492-3

Mazur-Panasiuk, N., Woźniakowski, G., Niemczuk, K., 2019. The first complete genomic sequences of African swine fever virus isolated in Poland. Scientific Reports 9, 1-9. https://doi.org/10. 1038/s41598-018-36823-0

Misinzo, G., Kasanga, C.J., Mpelumbe-Ngeleja, C., Masambu, J., Kitambi, A., Van Doorsselaere, J., 2012. African Swine Fever Virus, Tanzania, 2010-2012. Emerging Infectious Diseases 18, 2081-2083. https://doi.org/10.3201/eid1812.121083

Mwiine, F.N., Nkamwesiga, J., Ndekezi, C., Ochwo, S., 2019. Molecular Characterization of African Swine Fever Viruses from Outbreaks in Peri-Urban Kampala, Uganda. Advances in Virology 2019. https://doi.org/10.1155/2019/1463245

Ndlovu, S., Williamson, A.-L., Heath, L., Carulei, O., 2020a. Genome Sequences of Three African Swine Fever Viruses of Genotypes IV and XX from Zaire and South Africa, Isolated from a Domestic Pig (Sus scrofa domesticus), a Warthog (Phacochoerus africanus), and a European Wild Boar (Sus scrofa). Microbiology Resource Announcements 9.https://doi.org/10.1128/MRA.00341-20

Ndlovu, S., Williamson, A.-L., Malesa, R., Heerden, J. van, Boshoff, C.I., Bastos, A.D.S., Heath, L., Carulei, O., 2020b. Genome Sequences of Three African Swine Fever Viruses of Genotypes I, III, and XXII from South Africa and Zambia, Isolated from Ornithodoros Soft Ticks. Microbiology Resource Announcements 9.https://doi.org/10.1128/MRA.01376-19

Njau, E.P., Domelevo Entfellner, J.-B., Machuka, E.M., Bochere, E.N., Cleaveland, S., Shirima, G.M., Kusiluka, L.J., Upton, C., Bishop, R.P., Pelle, R., Okoth, E.A., 2021. The first genotype II African swine fever virus isolated in Africa provides insight into the current Eurasian pandemic. Sci. Rep. 11, 13081. https://doi.org/10. 1038/s41598-021-92593-2

Onzere, C.K., Bastos, A.D., Okoth, E.A., Lichoti, J.K., Bochere, E.N., Owido, M.G., Ndambuki, G., Bronsvoort, M., Bishop, R.P., 2018. Multi-locus sequence typing of African swine fever viruses from endemic regions of Kenya and Eastern Uganda (2011-2013) 
reveals rapid B602L central variable region evolution. Virus Genes 54, 111-123. https://doi.org/10.1007/s11262-017-1521-4

Penrith, M.L., Vosloo, W., 2009. Review of African swine fever: transmission, spread and control. Journal of the South African Veterinary Association 80, 58-62. https://doi.org/10.4102/jsava. v80i2.172

Penrith, M.-L., Bastos, A.D., Etter, E.M.C., Beltrán-Alcrudo, D., 2019. Epidemiology of African swine fever in Africa today: Sylvatic cycle versus socio-economic imperatives. Transboundary and Emerging Diseases 66, 672-686. https://doi.org/10.1111/tbed. 13117

Pikalo, J., Zani, L., Hühr, J., Beer, M., Blome, S., 2019. Pathogenesis of African swine fever in domestic pigs and European wild boar - Lessons learned from recent animal trials. Virus Research 271, 197614. https://doi.org/10.1016/j.virusres.2019.04.001

Quembo, C.J., Jori, F., Vosloo, W., Heath, L., 2018. Genetic characterization of African swine fever virus isolates from soft ticks at the wildlife/domestic interface in Mozambique and identification of a novel genotype. Transboundary and Emerging Diseases 65, 420-431. https://doi.org/10.1111/tbed.12700

Rodríguez, J.M., Moreno, L.T., Alejo, A., Lacasta, A., Rodríguez, F., Salas, M.L., 2015. Genome Sequence of African Swine Fever Virus BA71, the Virulent Parental Strain of the Nonpathogenic and Tissue-Culture Adapted BA71V. PLOS ONE 10, e0142889. https://doi.org/10.1371/journal.pone.0142889

Rowlands, R.J., Michaud, V., Heath, L., Hutchings, G., Oura, C., Vosloo, W., Dwarka, R., Onashvili, T., Albina, E., Dixon, L.K., 2008. African Swine Fever Virus Isolate, Georgia, 2007. Emerging Infectious Diseases 14, 1870-1874. https://doi.org/10.3201/ eid1412.080591

Sánchez, E.G., Pérez-Núñez, D., Revilla, Y., 2019. Development of vaccines against African swine fever virus. Virus Research 265, 150-155. https://doi.org/10.1016/j.virusres.2019.03.022

Tcherepanov, V., Ehlers, A., Upton, C., 2006. Genome Annotation Transfer Utility (GATU): rapid annotation of viral genomes using a closely related reference genome. BMC Genomics 7.https://doi. org/10.1186/1471-2164-7-150
Torresi, C., Fiori, M., Bertolotti, L., Floris, M., Colitti, B., Giammarioli, M., Giudici, S.D., Oggiano, A., Malmberg, M., Mia, G.M.D., Belák, S., Granberg, F., 2020. The evolution of African swine fever virus in Sardinia (1978-2014) as revealed by whole-genome sequencing and comparative analysis. Transboundary and Emerging Diseases 67, 1971-1980. https://doi.org/10.1111/tbed.13540

Urbano, A., Forth, J. h., Olesen, A. s., Dixon, L., Rasmussen, T. b., Cackett, G., Werner, F., Karger, A., Andrés, G., Wang, X., PerezNuñez, D., Galindo, I., Malogolovkin, A., Revilla, Y., Alonso, C., Gallardo, C., Blome, S., Arabyan, E., Zakaryan, H., Ferreira, F., 2021. 2. African swine fever virus: cellular and molecular aspects, in: Understanding and Combatting African Swine Fever. Wageningen Academic Publishers, pp. 25-61. https://doi.org/10.3920/ 978-90-8686-910-7_2

Yona, C.M., Vanhee, M., Simulundu, E., Makange, M., Nauwynck, H.J., Misinzo, G., 2020. Persistent domestic circulation of African swine fever virus in Tanzania, 2015-2017. BMC Veterinary Research 16, 369. https://doi.org/10.1186/s12917-020-02588-w

Zani, L., Forth, J.H., Forth, L., Nurmoja, I., Leidenberger, S., Henke, J., Carlson, J., Breidenstein, C., Viltrop, A., Höper, D., Sauter-Louis, C., Beer, M., Blome, S., 2018. Deletion at the 5'-end of Estonian ASFV strains associated with an attenuated phenotype. Scientific Reports 8.https://doi.org/10.1038/s41598-018-24740-1

Zhang, Z., Schwartz, S., Wagner, L., Miller, W., 2000. A greedy algorithm for aligning DNA sequences. Journal of Computational Biology 7, 203-214. https://doi.org/10.1089/10665270050081478

Zsak, L., Caler, E., Lu, Z., Kutish, G.F., Neilan, J.G., Rock, D.L., 1998. A Nonessential African Swine Fever Virus Gene UK Is a Significant Virulence Determinant in Domestic Swine. Journal of Virology $72,1028-1035$.

Publisher's note Springer Nature remains neutral with regard to jurisdictional claims in published maps and institutional affiliations. 